\title{
Linear differential equations for families of polynomials
}

Taekyun Kim" ${ }^{*}$, Dae San Kim², Toufik Mansour ${ }^{3}$ and Jong-Jin Seo ${ }^{4}$

"Correspondence: tkkim@kw.ac.kr 'Department of Mathematics, Kwangwoon University, Seoul, 139-701, South Korea

Full list of author information is available at the end of the article

\begin{abstract}
In this paper, we present linear differential equations for the generating functions of the Poisson-Charlier, actuarial, and Meixner polynomials. Also, we give an application for each case.
\end{abstract}

Keywords: actuarial polynomials; Meixner polynomials; Poisson-Charlier polynomials

\section{Introduction}

As is well known, the Poisson-Charlier polynomials $C_{k}(x ; a)$ are Sheffer sequences (see [1-4]) with $g(t)=e^{a\left(e^{t}-1\right)}$ and $f(t)=a\left(e^{t}-1\right)$, which are given by the generating function

$$
C(x, t)=e^{-t}(1+t / a)^{x}=\sum_{n \geq 0} C_{n}(x ; a) \frac{t^{n}}{n !} \quad(a \neq 0) .
$$

They satisfy the Sheffer identity

$$
C_{n}(x+y ; a)=\sum_{k=0}^{n}\left(\begin{array}{l}
n \\
k
\end{array}\right) a^{k-n} C_{k}(y ; a)(x)_{n-k},
$$

where $(x)_{n}$ is the falling factorial (see [5]). Moreover, these polynomials satisfy the recurrence relation

$$
C_{n+1}(x ; a)=a^{-1} x C_{n}(x-1 ; a)-C_{n}(x ; a) \quad(\text { see }[5]) .
$$

The first few polynomials are $C_{0}(x ; a)=1, C_{1}(x ; a)=-\frac{(a-x)}{a}, C_{2}(x ; a)=\frac{\left(a^{2}-x-2 a x+x^{2}\right)}{a^{2}}$.

The actuarial polynomials $a_{n}^{(\beta)}(x)$ are given by the generating function of Sheffer sequence

$$
F(x, t)=e^{\beta t+x\left(1-e^{t}\right)}=\sum_{n \geq 0} a_{n}^{(\beta)}(x) \frac{t^{n}}{n !} \quad(\text { see }[5]),
$$

and the Meixner polynomials of the first kind $m_{n}(x ; \beta, c)$ are also introduced in [5] as follows:

(c) $2016 \mathrm{Kim}$ et al. This article is distributed under the terms of the Creative Commons Attribution 4.0 International License (http://creativecommons.org/licenses/by/4.0/), which permits unrestricted use, distribution, and reproduction in any medium, provided you give appropriate credit to the original author(s) and the source, provide a link to the Creative Commons license, and indicate if changes were made. 


$$
M(x, t)=\sum_{n \geq 0} m_{n}(x ; \beta, c) \frac{t^{n}}{n !}=(1-t / c)^{x}(1-x)^{-x-\beta} .
$$

In mathematics, Meixner polynomials of the first kind (also called discrete Laguerre polynomials) are a family of discrete orthogonal polynomials introduced by Josef Meixner (see [6-10]). They are given in terms of binomial coefficients and the (rising) Pochhammer symbol by

$$
m_{n}(x, \beta, c)=\sum_{k=0}^{n}(-1)^{k}\left(\begin{array}{l}
n \\
k
\end{array}\right)\left(\begin{array}{l}
x \\
k
\end{array}\right) k !(x-\beta)_{n-k} c^{-k} \quad(\text { see }[5]) .
$$

Some interesting identities and properties of the Poisson-Charlier, actuarial, and Meixner polynomials can be derived from umbral calculus (see [11-13]). Kim and Kim [12] introduced nonlinear Changhee differential equations for giving special functions and polynomials. Many researchers have studied the Poisson-Charlier, actuarial and Meixner polynomials in the mathematical physics, combinatorics, and other applied mathematics (for example, see $[14,15])$.

In this paper, we study linear differential equations arising from the Poisson-Charlier, actuarial, and Meixner polynomials and derive new recurrence relations for those polynomials from our differential equations.

\section{Poisson-Charlier polynomials}

Recall that the falling polynomials $(x)_{N}$ are defined by $(x)_{N}=(x-1) \cdots(x-N+1)$ for $N \geq 1$ with $(x)_{0}=1$. For brevity, we denote the generating functions $C(x, t)$ and $\frac{d^{j}}{d t} C(x ; t)$ by $C$ and $C^{(j)}$ for $j \geq 0$.

Lemma 1 The generating function $C^{(N)}$ is given by $\left(\sum_{i=0}^{N} a_{i}(N, x)(t+a)^{-i}\right) C$, where $a_{0}(N, x)=(-1)^{N}, a_{N}(N, x)=(x)_{N}$, and

$$
a_{i}(N, x)=(x-i+1) a_{i-1}(N-1, x)-a_{i}(N-1, x) \quad(1 \leq i \leq N-1) .
$$

Proof Clearly, $a_{0}(0, x)=1$. For $N=1$, by (1) we have $C^{(1)}=\left(-1+x(t+a)^{-1}\right) C$, which proves the lemma for $N=1$ (here $a_{0}(1, x)=-1$ and $a_{1}(1, x)=x$ ). Assume that $C^{(N)}$ is given by $\left(\sum_{i=0}^{N} a_{i}(N, x)(t+a)^{-i}\right) C$. Then

$$
\begin{aligned}
C^{(N+1)} & =\left(-\sum_{i=0}^{N} a_{i}(N, x) i(t+a)^{-i-1}\right) C+\left(\sum_{i=0}^{N} a_{i}(N, x)(t+a)^{-i}\right)\left(-1+x(t+a)^{-1}\right) C \\
& =\left(\sum_{i=1}^{N+1}(x-i+1) a_{i-1}(N, x)(t+a)^{-i}-\sum_{i=0}^{N} a_{i}(N, x)(t+a)^{-i}\right) C .
\end{aligned}
$$

This shows that the generating function $C^{(N+1)}$ is given by

$$
\begin{aligned}
& \left(-a_{0}(N, x)+\sum_{i=1}^{N}\left((x-i+1) a_{i-1}(N, x)-a_{i}(N, x)\right)(t+a)^{-i}\right. \\
& \left.+(x-N) a_{N}(N, x)(t+a)^{-N-1}\right) C .
\end{aligned}
$$

Comparing with $C^{(N+1)}=\left(\sum_{i=0}^{N+1} a_{i}(N+1, x)(t+a)^{-i}\right) C$, we complete the proof. 
In order to obtain an explicit formula for the generating function $C^{(N)}$, we need the following lemma.

Lemma 2 For all $0 \leq i \leq N$, the coefficient's $a_{i}(N, x)$ in Lemma 1 are given by

$$
a_{i}(N, x)=(x)_{i}\left(\begin{array}{c}
N \\
i
\end{array}\right)(-1)^{N-i}
$$

Proof By Lemma 1 we have that

$$
a_{i}(N+1, x)=(x-i+1) a_{i-1}(N, x)-a_{i}(N, x), \quad 0 \leq i \leq N+1,
$$

with $a_{0}(0, x)=1$ and $a_{i}(N, x)=0$ whenever $i>N$ or $i<0$. Define $A_{i}(x ; t)=\sum_{N \geq i} a_{i}(N, x) t^{N}$. Then we have

$$
A_{i}(x ; t)=\frac{(x+1-i) t}{1+t} A_{i-1}(x)
$$

with $A_{0}(x ; t)=\frac{1}{1+t}$. By induction on $i$ we derive that $A_{i}(x, t)=\frac{(x)_{i} t^{i}}{(1+t)^{i+1}}$. Hence, by the fact that $\frac{1}{(1+t)^{i+1}}=\sum_{j \geq 0}\left(\begin{array}{c}i+j \\ i\end{array}\right)(-1)^{j} t^{j}$ we obtain that $a_{i}(N, x)=(x)_{i}\left(\begin{array}{c}N \\ i\end{array}\right)(-1)^{N-i}$, as required.

Thus, by Lemmas 1 and 2 we can state the following result.

Theorem 3 The linear differential equations

$$
C^{(N)}=\left(\sum_{i=0}^{N}(x)_{i}\left(\begin{array}{c}
N \\
i
\end{array}\right)(-1)^{N-i}(t+a)^{-i}\right) C \quad(n=0,1, \ldots)
$$

have a solution $C(x, t)=e^{-t}(1+t / a)^{x}$, where $(x)_{i}=x(x-1) \cdots(x+1-i)$ with $(x)_{0}=1$.

As an application of Theorem 3, we obtain the following corollary.

Corollary 4 For all $k, N \geq 0$,

$$
C_{k+N}(x ; a)=\sum_{i=0}^{N} \sum_{m=0}^{k}(x)_{i}\left(\begin{array}{c}
N \\
i
\end{array}\right)\left(\begin{array}{c}
k \\
m
\end{array}\right)(-1)^{N-i+m}(i+m-1)_{m} a^{-i-m} C_{k-m}(x ; a) .
$$

Proof By (1) and Theorem 3 we have

$$
C^{(N)}=\left(\sum_{i=0}^{N}(x)_{i}\left(\begin{array}{c}
N \\
i
\end{array}\right)(-1)^{N-i}(t+a)^{-i}\right) \sum_{\ell \geq 0} C_{\ell}(x ; a) \frac{t^{\ell}}{\ell !} .
$$

Since $\frac{1}{(1+t)^{i+1}}=\sum_{j \geq 0}\left(\begin{array}{c}i+j \\ i\end{array}\right)(-1)^{j} t^{j}$, we obtain

$$
C^{(N)}=\sum_{k \geq 0} \sum_{i=0}^{N} \sum_{m=0}^{k}(x)_{i}\left(\begin{array}{c}
N \\
i
\end{array}\right)\left(\begin{array}{c}
k \\
m
\end{array}\right)(-1)^{N-i+m}(i+m-1)_{m} a^{-i-m} C_{k-m}(x ; a) \frac{t^{k}}{k !} .
$$

By comparing coefficients of $t^{k}$ we complete the proof. 


\section{Actuarial polynomials}

For brevity, we denote the generating functions $F(x, t)=e^{\beta t+x\left(1-e^{t}\right)}$ and $\frac{d^{j}}{d t^{j}} F(x ; t)$ by $F$ and $F^{(j)}$ for $j \geq 0$.

Lemma 5 The generatingfunction $F^{(N)}$ is given by $\left(\sum_{i=0}^{N} b_{i}(N, x) e^{i t}\right) F$, where $b_{0}(N, x)=\beta^{N}$, $b_{N}(N, x)=(-x)^{N}$, and $b_{i}(N, x)=-x b_{i-1}(N-1, x)+(\beta+i) b_{i}(N-1, x)(1 \leq i \leq N-1)$.

Proof Clearly, $b_{0}(0, x)=1$. For $N=1$, by (2) we have $F^{(1)}=\left(\beta-x e^{t}\right) F$, which proves the lemma for $N=1$ (here $b_{0}(1, x)=\beta$ and $\left.b_{1}(1, x)=-x\right)$. Assume that $F^{(N)}$ is given by $\left(\sum_{i=0}^{N} b_{i}(N, x) e^{i t}\right) F$. Then

$$
\begin{aligned}
F^{(N+1)} & =\left(\sum_{i=0}^{N} b_{i}(N, x) i e^{i t}\right) F+\left(\sum_{i=0}^{N} b_{i}(N, x) e^{i t}\right)\left(\beta-x e^{t}\right) F \\
& =\left(\sum_{i=0}^{N}(\beta+i) a_{i}(N, x) e^{i t}-x \sum_{i=1}^{N+1} b_{i-1}(N, x) e^{i t}\right) F,
\end{aligned}
$$

which shows that the generating function $F^{(N+1)}$ is given by

$$
\left(\beta b_{0}(N, x)+\sum_{i=1}^{N}\left(-x a_{i-1}(N, x)+(\beta+i) b_{i}(N, x)\right) e^{i t}-x b_{N}(N, x) e^{(N+1) t}\right) F .
$$

Comparing with $F^{(N+1)}=\left(\sum_{i=0}^{N+1} b_{i}(N+1, x) e^{i t}\right) C$, we complete the proof.

Lemma 6 For all $0 \leq i \leq N$, the coefficients $b_{i}(N, x)$ in Lemma 5 are given by

$$
b_{i}(N, x)=(-x)^{i} \sum_{j=i}^{N}\left(\begin{array}{c}
N \\
j
\end{array}\right) \beta^{N-j} S(j, i)
$$

where $S(n, k)$ are the Stirling numbers (for example, see [16]) of the second kind.

Proof By Lemma 5 we have that

$$
b_{i}(N+1, x)=-x b_{i-1}(N, x)+(\beta+i) b_{i}(N, x), \quad 0 \leq i \leq N+1,
$$

with $b_{0}(0, x)=1$ and $b_{i}(N, x)=0$ whenever $i>N$ or $i<0$. Define $B_{i}(x ; t)=\sum_{N \geq i} b_{i}(N, x) t^{N}$. Then we have

$$
B_{i}(x ; t)=\frac{-x t}{1-(\beta+i) t} B_{i-1}(x)
$$

with $B_{0}(x ; t)=\frac{1}{1-\beta t}$. By induction on $i$ we derive that

$$
B_{i}(x, t)=\frac{(-x t)^{i}}{(1-\beta t)(1-(\beta+1) t) \cdots(1-(\beta+i) t)}=\frac{(-x t)^{i}}{(1-\beta t)^{i+1}} \prod_{j=0}^{i} \frac{1}{1-j t /(1-\beta t)} .
$$


Hence, since $\frac{x^{k}}{(1-x)(1-2 x) \cdots(1-k x)}=\sum_{n \geq k} S(n, k) x^{n}$ (for example, see [16]), where $S(n, k)$ are the Stirling numbers of the second kind, we obtain that

$$
B_{i}(x, t)=(-x)^{i} \sum_{j \geq i} S(j, i) \frac{t^{j}}{(1-\beta t)^{j+1}}
$$

Since $\frac{1}{(1+t)^{i+1}}=\sum_{j \geq 0}\left(\begin{array}{c}i+j \\ i\end{array}\right)(-1)^{j} t^{j}$, we obtain that

$$
B_{i}(x, t)=(-x)^{i} \sum_{j \geq i} \sum_{\ell \geq 0}\left(\begin{array}{c}
j+\ell \\
j
\end{array}\right) \beta^{\ell} S(j, i) t^{J+\ell}
$$

Thus, by finding the coefficients of $t^{N}$ we complete the proof.

Thus, by Lemmas 5 and 6 we can state the following result.

Theorem 7 The linear differential equations

$$
F^{(N)}=\sum_{i=0}^{N}\left((-x)^{i} e^{i t} \sum_{j=i}^{N}\left(\begin{array}{c}
N-1 \\
j-1
\end{array}\right) \beta^{N-j} S(j, i)\right) F \quad(N=0,1, \ldots)
$$

have a solution $F(x, t)=e^{\beta t+x\left(1-e^{t}\right)}$.

Recall that $F(x, t)=e^{\beta t+x\left(1-e^{t}\right)}=\sum_{n \geq 0} a_{n}^{(\beta)}(x) \frac{t^{n}}{n !}$, which is the generating function for the actuarial polynomials $a_{n}^{(\beta)}(x)$ (see (2)). As an application of Theorem 7 , we obtain the following corollary.

Corollary 8 For all $k, N \geq 0$,

$$
a_{N+k}^{(\beta)}(x)=\sum_{i=0}^{N} \sum_{m=0}^{k} b_{i}(N ; x)\left(\begin{array}{l}
k \\
m
\end{array}\right) i^{k-m} a_{m}^{(\beta)}(x),
$$

where $b_{i}(N, x)=(-x)^{i} \sum_{j=i}^{N}\left(\begin{array}{c}N-1 \\ j-1\end{array}\right) \beta^{N-j} S(j, i)$.

Proof By (2) and Theorem 7 we have $F^{(N)}=\left(\sum_{i=0}^{N} b_{i}(N, x) e^{i t}\right) \sum_{\ell \geq 0} a_{\ell}^{(\beta)}(x) \frac{t^{\ell}}{\ell !}$. Thus,

$$
F^{(N)}=\sum_{k \geq 0} \sum_{i=0}^{N} \sum_{m=0}^{k} b_{i}(N, x)\left(\begin{array}{c}
k \\
m
\end{array}\right) i^{k-m} a_{m}^{(\beta)}(x) \frac{t^{k}}{k !} .
$$

By comparing the coefficients of $t^{N+k}$ we complete the proof.

\section{Meixner polynomials of the first kind}

Recall that the rising polynomials $\langle x\rangle_{N}$ are defined by $\langle x\rangle_{N}=x(x+1) \cdots(x+N-1)$ with $\langle x\rangle_{0}=1$. For brevity, we denote the generating functions $M(x, t)=(1-t / c)^{x}(1-x)^{-x-\beta}$ and $\frac{d^{j}}{d t} M(x ; t)$ by $M$ and $M^{(j)}$ for $j \geq 0$, respectively. 
Theorem 9 The linear differential equations

$$
M^{(N)}=\left(\sum_{i=0}^{N}(-1)^{i}\left(\begin{array}{c}
N \\
i
\end{array}\right)(x)_{N-i}(x+\beta\rangle_{i}(t-1)^{-i}(t-c)^{-(N-i)}\right) M \quad(N=0,1, \ldots)
$$

have a solution $M=M(x, t)=(1-t / c)^{x}(1-x)^{-x-\beta}$.

Proof We proceed the proof by induction on $N$. Clearly, the theorem holds for $N=0$. By (3) we have $M^{(1)}=\left(x(t-c)^{-1}-(x+\beta)(t-1)^{-1}\right) M$, which proves the theorem for $N=1$. Assume that the theorem holds for $N \geq 1$. Then by the induction hypothesis we have

$$
\begin{aligned}
& M^{(N+1)} \\
& =\frac{d}{d t}\left(\sum_{i=0}^{N}(-1)^{i}\left(\begin{array}{c}
N \\
i
\end{array}\right)(x)_{N-i}\langle x+\beta\rangle_{i}(t-1)^{-i}(t-c)^{-(N-i)}\right) M \\
& =\left\{\left(\sum_{i=0}^{N}(-1)^{i+1} i\left(\begin{array}{c}
N \\
i
\end{array}\right)(x)_{N-i}\langle x+\beta\rangle_{i}(t-1)^{-i-1}(t-c)^{-(N-i)}\right) M\right. \\
& +\left(\sum_{i=0}^{N}(-1)^{i+1}(N-i)\left(\begin{array}{c}
N \\
i
\end{array}\right)(x)_{N-i}\langle x+\beta\rangle_{i}(t-1)^{-i}(t-c)^{-(N+1-i)}\right) M \\
& +\left(\sum_{i=0}^{N}(-1)^{i}\left(\begin{array}{c}
N \\
i
\end{array}\right)(x)_{N-i}\langle x+\beta\rangle_{i}(t-1)^{-i}(t-c)^{-(N-i)}\right) \\
& \left.\quad \times\left(x(t-c)^{-1}-(x+\beta)(t-1)^{-1}\right) M\right\} .
\end{aligned}
$$

After rearranging the indices of the sums, we obtain

$$
\begin{aligned}
M^{(N+1)} & \\
= & \left(\sum_{i=1}^{N+1}(-1)^{i}(i-1)\left(\begin{array}{c}
N \\
i-1
\end{array}\right)(x)_{N+1-i}\langle x+\beta\rangle_{i-1}(t-1)^{-i}(t-c)^{-(N+1-i)}\right) M \\
& +\left(\sum_{i=0}^{N}(-1)^{i+1}(N-i)\left(\begin{array}{c}
N \\
i
\end{array}\right)(x)_{N-i}\langle x+\beta\rangle_{i}(t-1)^{-i}(t-c)^{-(N+1-i)}\right) M \\
& +\left(\sum_{i=0}^{N}(-1)^{i}\left(\begin{array}{c}
N \\
i
\end{array}\right) x(x)_{N-i}\langle x+\beta\rangle_{i}(t-1)^{-i}(t-c)^{-(N+1-i)}\right) M \\
& +\left(\sum_{i=1}^{N+1}(-1)^{i}\left(\begin{array}{c}
N \\
i-1
\end{array}\right)(x)_{N+1-i}(x+\beta)\langle x+\beta\rangle_{i-1}(t-1)^{-i}(t-c)^{-(N+1-i)}\right) M .
\end{aligned}
$$

This implies

$$
M^{(N+1)}=\left(\sum_{i=0}^{N+1}(-1)^{i}\left(\begin{array}{c}
N+1 \\
i
\end{array}\right)(x)_{N+1-i}\langle x+\beta\rangle_{i}(t-1)^{-i}(t-c)^{-(N+1-i)}\right) M,
$$

and the induction step is completed. 
From (3) we have $M^{(N)}=\sum_{k \geq 0} m_{k+N}(x ; \beta, c) \frac{t^{k}}{k !}$ for all $N \geq 0$. Similarly to the previous section, we have a recurrence relation for the coefficients of $m_{n}(x ; \beta, c)$.

Corollary 10 For all $k, N \geq 0$,

$$
m_{k+N}(x ; \beta, c)=(-1)^{N} \sum_{i=0}^{N}(-1)^{i}\left(\begin{array}{c}
N \\
i
\end{array}\right)(x)_{N-i}\langle x+\beta\rangle_{i} \sum_{\ell+m+n=k} \frac{k !\left(\begin{array}{c}
i+\ell-1 \\
\ell
\end{array}\right)\left(\begin{array}{c}
N+m-i-1 \\
m
\end{array}\right)}{n ! c^{N-i+m}} m_{n}(x ; \beta, c) .
$$

Proof By Theorem 9 we have

$$
M^{(N)}=\left(\sum_{i=0}^{N}(-1)^{i}\left(\begin{array}{c}
N \\
i
\end{array}\right)(x)_{N-i}\langle x+\beta\rangle_{i}(t-1)^{-i}(t-c)^{-(N-i)}\right) \sum_{\ell \geq 0} m_{\ell}(x ; \beta, c) \frac{t^{\ell}}{\ell !} .
$$

Thus, since $(t-c)^{-s}=(-1)^{s} \sum_{\ell \geq 0}\left(\begin{array}{c}s+\ell-1 \\ \ell\end{array}\right) c^{-s-\ell} t^{\ell}$, we obtain

$$
\begin{aligned}
M^{(N)}= & (-1)^{N} \sum_{i=0}^{N}(-1)^{i}\left(\begin{array}{c}
N \\
i
\end{array}\right)(x)_{N-i}\langle x+\beta\rangle_{i} \\
& \times \sum_{\ell \geq 0} \sum_{m \geq 0} \sum_{n \geq 0}\left(\begin{array}{c}
i+\ell-1 \\
\ell
\end{array}\right)\left(\begin{array}{c}
N+m-i-1 \\
m
\end{array}\right) m_{n}(x ; \beta, c) \frac{c^{-N-m+i} t^{\ell+m+n}}{n !} .
\end{aligned}
$$

Hence, by finding the coefficients of $t^{k}$ in the generating function $M^{(N)}$ we complete the proof.

\section{Results and discussion}

In this paper, the Poisson-Charlier polynomials, actuarial, and Meixner polynomial are introduced. We study linear differential equations arising from the Poisson-Charlier, actuarial, and Meixner polynomials and present some their recurrence relations. Linear differential equations for various families of polynomials are derived. Furthermore, some particular cases of the results are presented.

\section{Competing interests}

The authors declare that they have no competing interests.

\section{Authors' contributions}

All authors contributed equally to the manuscript and typed, read, and approved the final manuscript.

\section{Author details}

'Department of Mathematics, Kwangwoon University, Seoul, 139-701, South Korea. ${ }^{2}$ Department of Mathematics, Sogang University, Seoul, 121-742, South Korea. ${ }^{3}$ Department of Mathematics, University of Haifa, Haifa, 3498838, Israel. ${ }^{4}$ Department of Applied Mathematics, Pukyong National University, Busan, 48513, South Korea.

\section{Acknowledgements}

The present research has been conducted by the Research Grant of Kwangwoon University in 2016.

Received: 26 January 2016 Accepted: 10 March 2016 Published online: 22 March 2016

\section{References}

1. Dieulefait, C: On the Poisson-Charlier series. An. Soc. Ci. Argentina 128, 10-24 (1939) (in Spanish)

2. Karadzhov, GE: Spectral asymptotics for Toeplitz matrices generated by the Poisson-Charlier polynomials. Proc. Am. Math. Soc. 114(1), 129-134 (1992)

3. Khan, MA: On some new generating functions for Poisson-Charlier polynomials of several variables. Math. Sci. Res. J. 15(5), 127-136 (2011)

4. Roman, S: The Umbral Calculus. Pure and Applied Mathematics, vol. 111. Academic Press [Harcourt Brace Jovanovich, Publishers], New York (1984) 
5. Pender, J: A Poisson-Charlier approximation for nonstationary queues. Oper. Res. Lett. 42(4), $293-298$ (2014)

6. Atakishiyev, NM, Jafarova, AM, Jafarov, El: Meixner polynomials and representations of the 3D Lorentz group SO(2,1). Ann. Math. Stat. 17(2), 14-23 (2014)

7. Dominici, D: Mehler-Heine type formulas for Charlier and Meixner polynomials. Ramanujan J. 39(2), $271-289$ (2016)

8. Gaboriaud, J, Genest, VX, Lemieux, J, Vinet, L: A superintegrable discrete oscillator and two-variable Meixner polynomials. J. Phys. A 48(41), 415202 (2015)

9. Kruchinin, DV, Shablya, YV: Explicit formulas for Meixner polynomials. Int. J. Math. Math. Sci. 2015, Article ID 620569 (2015)

10. Miki, H, Tsujimoto, S, Vinet, L, Zhedanov, A: An algebraic model for the multiple Meixner polynomials of the first kind. J. Phys. A 45(32), 325205 (2012)

11. Kim, DS, Kim, T, Kwon, HI, Mansour, T: Barnes-type Narumi of the second kind and Poisson-Charlier mixed-type polynomials. J. Comput. Anal. Appl. 19(5), 837-850 (2015)

12. Kim, DS, Kim, T: A note on nonlinear Changhee differential equations. Russ. J. Math. Phys. (to appear)

13. Kim, T: Identities involving Laguerre polynomials derived from umbral calculus. Russ. J. Math. Phys. 21(1), 36-45 (2014)

14. Aptekarev, Al, Tulyakov, DN: The saturation regime of Meixner polynomials and the discrete Bessel kernel. Mat. Zametki 98(1), 147-151 (2015) (in Russian)

15. Truesdell, C: A note on the Poisson-Charlier functions. Ann. Math. Stat. 18, 450-454 (1947)

16. Mansour, T, Schork, M: Commutation Relations, Normal Ordering and Stirling Numbers. Chapman \& Hall/CRC, Boca Raton (2015)

\section{Submit your manuscript to a SpringerOpen ${ }^{\circ}$ journal and benefit from:}

- Convenient online submission

Rigorous peer review

- Immediate publication on acceptance

- Open access: articles freely available online

- High visibility within the field

- Retaining the copyright to your article 